\title{
Può la traduzione automatica favorire il plurilinguismo nell'Unione europea post-Brexit?
}

\author{
Michele Gazzola
}

PUBBLICATO: 26 LUGLIO 2021

\section{Introduzione}

I 124 febbraio 2020 venne pubblicato sul sito dell'Unione Europea (UE) un comunicato ufficiale destinato al grande pubblico in cui si illustravano i provvedimenti urgenti adottati dalla Commissione europea in materia di coronavirus. Si trattava di un testo di circa quattro pagine che presentava le iniziative scientifiche, organizzative e finanziarie messe in atto nelle settimane precedenti per affrontare l'esplosione dell'epidemia da Covidıg, la quale in quel momento stava interessando principalmente l'Italia. Eppure il comunicato era disponibile solo in lingua inglese. Le traduzioni nelle altre lingue arrivarono giorni dopo. Quelle in italiano, in quel momento probabilmente le più urgenti, furono pubblicate dopo quelle in maltese e spagnolo (Di Stefano 2020). I servizi di traduzione dell'Unione, i più possenti al mondo come risorse e numero di funzionari impiegati, non furono attivati per pubblicare contemporaneamente informazioni importanti nelle 24 lingue ufficiali dell'Unione. La mancanza di traduzioni tempestive si tradusse in un'ineguaglianza sostanziale nell'accesso a importanti informazioni in materia di tutela dalla salute pubblica.

Non si è trattato di un caso isolato; un altro recente esempio riguarda la sfera dell'accesso ai finanziamenti europei. Il 25 marzo del 202I la Commissione europea ha pubblicato sul proprio sito Internet la guida al nuovo programma Erasmus+, il nuovo programma quadro dell'Unione europea per l'istruzione, la formazione, la gioventù e lo sport per il periodo 202I-2027. Lo stanziamento complessivo per il programma Erasmus+ è di 26,2 miliardi di euro. Erasmus+ è un programma aperto non solo agli specialisti, ma anche a grandi e piccole associazioni, enti pubblici e organizzazioni non governative. Il manuale del programma consta di 328 pagine che descrivono nel dettaglio le complesse procedure per ottenere il sostegno finanziario dell'UE. All'inaugurazione del programma il suddetto manuale era disponibile solo in inglese, lingua che dopo l'uscita dal Regno Unito dall'Unione è parlata come lingua materna da circa l's\% della popolazione europea. Le traduzioni nelle altre lingue ufficiali dell'UE sono arrivate circa tre settimane dopo, anche se la prima scadenza per presentare proposte di progetti era già l'is maggio 202r. In pratica le associazioni anglofone o dotate di personale fluente in inglese hanno avuto il doppio del tempo per prepararsi alla prima scadenza rispetto ai concorrenti. I ritardi sulle traduzioni sono stati oggetto di alcune interrogazioni parlamentari urgenti.

Né le pagine informative sul Covidıg né il manuale del programma Erasmus+ sono pubblicazioni soggette al Regolamento numero I del 1958 (da qui in poi Regolamento n. I/58), il testo che, come vedremo nel prossimo paragrafo, disciplina l'uso delle lingue nell'Unione. Ma il Regolamento i/58 è un testo scritto in un'epoca in cui la comunicazione digitale non esisteva ancora. In mancanza di un aggiornamento di un testo ormai datato, si è creato nel tempo uno spazio di incertezza normativa nel quale le istituzioni europee possono perseguire una politica linguistica del fatto compiuto che in alcuni ambiti finisce per promuovere surrettiziamente la preminenza di una o poche lingue sulle altre, e questo anche in situazioni in cui una comunicazione plurilingue sarebbe più efficace. I due aneddoti citati nell'introduzione sono per l'appunto aneddoti, ma rivelano una tendenza generale. I risultati di uno studio pilota realizzato nel 2014 sulle pagine di entrata di tutte le Direzioni Generali (DG) della 
Commissione europea, infatti, mostrano che I4 DG su 33 avevano pubblicato le loro pagine di entrata solo in inglese, otto DG in inglese, francese e tedesco, una DG in undici lingue e dieci DG in 24 o 23 lingue ufficiali (Gazzola 2014).

Questo articolo riesamina la questione della politica linguistica dell'Unione europea alla luce di due importanti novità: l'irreversibile rivoluzione digitale in corso - accelerata e favorita dalle restrizioni alla mobilità e al contatto interpersonale causate dall'epidemia da Covidrs -, e l'uscita del Regno Unito dall'Unione europea formalizzata il 3I gennaio 2020 e perfezionata il 3I dicembre 2020 al termine di un periodo transitorio.

\section{Tensioni e contraddizioni della politica linguistica dell'Unione europea}

La politica linguistica dell'UE comprende diversi ambiti di interesse, tra cui vanno segnalati l'apprendimento delle lingue straniere, l'integrazione linguistica dei migranti e il sostegno alle lingue minoritarie tradizionali ${ }^{2}$. Il suo nucleo è tuttavia la gestione della comunicazione plurilingue dell'UE stessa. Questo ambito della politica linguistica è centrale perché riguarda direttamente i rapporti fra le istituzioni dell'UE da un lato, e i cittadini e gli Stati membri dall'altro lato. L'utilizzo di più lingue nella comunicazione ufficiale consente ai cittadini di comprendere la natura delle istituzioni europee e le politiche della UE, e garantisce al tempo stesso l'equità di accesso alle opportunità finanziamento e di lavoro dell'Unione. La politica linguistica istituzionale è anche l'unica ad essere sotto il diretto controllo dell'Unione, mentre negli altri campi di intervento sopra menzionati la UE opera per lo più indirettamente attraverso raccomandazioni, attività di coordinamento tra paesi o finanziamenti.

La politica linguistica istituzionale di unorganizzazione è chiamata regime linguistico. Esso consiste nel definire un insieme di lingue ufficiali e di lavoro, un insieme di regole riguardanti il loro utilizzo nella comunicazione interna allorganizzazione e nella comunicazione rivolta al pubblico, e il ruolo dei servizi di mediazione linguistica quali la traduzione e l'interpretariato. La storia e la struttura del regime linguistico dell'UE non possono essere trattate dettagliatamente qui3. Basti ricordare che il primissimo regolamento adottato dalle allora Comunità europee (appunto il Regolamento n. I/58) riguardava per l'appunto il regime linguistico. Questo era inevitabile poiché le organizzazioni internazionali devono prima accordarsi sulle lingue in cui lavorare prima di poter iniziare ad essere operative.

Il Regolamento n. I/58, arricchitosi di nuove lingue nel tempo in seguito ai vari allargamenti dell'Unione, stabilisce che l'UE ha 24 lingue ufficiali e di lavoro, e che queste lingue devono essere usate nelle comunicazioni da e verso gli Stati membri e i cittadini, nei regolamenti e negli atti di applicazione generale, e nella pubblicazione della Gazzetta ufficiale dell'Unione europea. Una certa flessibilità è invece prevista per l'applicazione del regolamento all'interno delle singole istituzioni. Il Regolamento n. I/58 può essere cambiato solo all'unanimità dal Consiglio dell'Unione europea. Va inoltre aggiunto che dall'entrata in vigore del trattato di Lisbona nel 2009, i cittadini dell'Unione hanno il diritto di presentare petizioni al Parlamento europeo, di rivolgersi al Mediatore europeo e alle istituzioni e agli organi consultivi dell'Unione in una delle lingue ufficiali e di ricevere una risposta nella stessa lingua.

Il Regolamento n. I/58 non esige che ogni singolo documento, testo pubblicato e discorso sia tradotto o interpretato in tutte le lingue ufficiali. Osserviamo quindi un'ampia gamma di prassi e di regimi linguistici de facto (al plurale). In alcuni contesti, come le sedute plenarie del Parlamento europeo, vige un regime completamente plurilingue in cui la traduzione e l'interpretazione sono 
assicurate da e verso tutte le lingue ufficiali; in altre riunioni vige invece un sistema asimmetrico, vale a dire un sistema in cui è possibile parlare o scrivere in diverse lingue, ma l'interpretazione e la traduzione sono assicurate solo in un numero ristretto di idiomi. Altre istituzioni hanno adottato in pratica una o poche lingue di lavoro nella comunicazione interna. Il francese è la lingua esclusiva per le deliberazioni della Corte di giustizia, mentre l'inglese è la principale lingua di lavoro della Banca centrale europea. Diversi documenti prodotti dalla Commissione che non sono giuridicamente vincolanti, come alcuni documenti di lavoro, molte relazioni o pagine Internet, sono disponibili solo in alcune lingue o in una sola, di solito in inglese e talvolta in francese.

Al di là degli aspetti specifici dei regimi linguistici affermatisi nella prassi, ciò che occorre qui sottolineare è che la gestione della diversità linguistica europea presenta diverse contraddizioni e tensioni. Le disposizioni dei trattati e del Regolamento n. I/58 lasciano ampi margini di discrezionalità, ma questo rischia di creare delle situazioni in cui la vaghezza delle regole formali generali e il potere delle consuetudini finiscono per generare forme di disuguaglianza sostanziale che casi estremi possono configurarsi come vere e proprie discriminazioni. Se i vincoli di tempo e di bilancio non possono essere ignorati, è allo stesso tempo importante ridurre al minimo le disuguaglianze fra cittadini e attori portatori di interesse nell'accesso ai documenti europei ${ }^{4}$.

La distinzione tra atto giuridicamente vincolante, o atto che crea diritti e obblighi, da un lato, e atto non giuridicamente vincolante, dall'altro, è certamente di fondamentale importanza. L'applicabilità diretta e l'efficacia del diritto dell'Unione non possono funzionare in un contesto di diritto UE non plurilingue (Ziller 2020). Come ricordato dalla Corte di Giustizia europea nella sentenza del II dicembre 2007 Skoma-Lux, Causa C-I6I/o6 (punto 30):

il principio fondamentale della certezza del diritto impone che una normativa comunitaria consenta agli interessati di conoscere esattamente la portata degli obblighi che essa prescrive loro, ciò che può essere garantito esclusivamente dalla regolare pubblicazione della suddetta normativa nella lingua ufficiale del destinatario.

Esiste tuttavia una 'zona grigia', ovvero una zona di incertezza non direttamente coperta dal Regolamento n. I/58, nella quale le istituzioni, gli organi e le agenzie europee pubblicano documenti o linee guida che, pur non essendo disponibili nella Gazzetta Ufficiale, sono di fatto giuridicamente vincolanti, oppure prendono decisioni con effetti sostanziali sugli interessi dei cittadini e delle impese che potrebbero essere sottoposte all'obbligo di traduzione. Oltre al caso di Erasmus+ cui si accennato nell'introduzione di questo articolo, un esempio recente è quello della comunicazione nell'ambito degli aiuti di Stato; molti comunicati stampa e diverse dichiarazioni della Commissione europea riguardo il "Quadro temporaneo per le misure di aiuto di Stato a sostegno dell'economia nell'attuale emergenza del COVID-ı9", per esempio, sono disponibili solo in inglese5.

\section{La sfida della rivoluzione digitale}

Le trasformazioni tecnologiche avvenute negli ultimi due decenni hanno di fatto sfumato i confini tra comunicazione interna a un'organizzazione e comunicazione rivolta al pubblico. In primo luogo, l'uso sempre più pervasivo delle tecnologie digitali fa sì che molti documenti interni come i dibattiti orali nelle sedute del Parlamento europeo, i tweet, le bozze di documenti di lavoro, i video o i messaggi multimodali siano diventati pubblici. In secondo luogo, la rivoluzione digitale ha reso sempre meno importante la distinzione fra documenti scritti e documenti orali. Molti testi scritti sono divenuti liquidi; le pagine Internet di una organizzazione possono essere continuamente modificate, 
aggiornate e rimosse senza lasciare tracce. I documenti orali come i discorsi dei commissari UE e le sedute del Parlamento europeo sono invece diventati 'solidi', nel senso che essi sono accessibili via streaming e sono registrabili su semplici supporti video che possono essere facilmente archiviati e consultati da qualsiasi parte del mondo.

L'importanza di garantire il plurilinguismo nelle pagine dei siti Internet delle istituzioni dell'Unione europea, e non solo nelle pubblicazioni nella Gazzetta Ufficiale, è stata ricordata e sottolineata dal Consiglio dell'Unione europea nel 2008 nella Risoluzione del 21 novembre 2008 relative a una strategia europea per il multilinguismo (GU C 320 del I6.I2.2008, pagg. I-3). In questa risoluzione, il Consiglio ha invitato la Commissione

ad adottare misure intese a tenere in debito conto le esigenze linguistiche dei cittadini e delle istituzioni, prestando particolare attenzione (i) alle relazioni tra le istituzioni europee e il pubblico, (ii) alle relazioni tra le istituzioni europee e le istituzioni nazionali, e provvedendo in particolare a fornire ai cittadini informazioni in tutte le lingue ufficiali e a promuovere il multilinguismo sui siti web della Commissione.

Più recentemente, la questione della mancanza di plurilinguismo nelle pagine in rete è stata portata alla ribalta in una consultazione pubblica del Mediatore europeo sul multilinguismo nell'Unione europea (Mediatore europeo 2019). I rischi di perdita della diversità linguistica e le potenziali disuguaglianze nel mondo digitale sono stati recentemente sollevati nella Risoluzione del Parlamento europeo dell'11 settembre 2018 sull'uguaglianza linguistica nell'era digitale (2018/2028(INI)).

\section{Il mito dell'inglese "ingua franca"}

La politica linguistica adottata dalla Commissione per il proprio sito Internet dà esplicita priorità alle pubblicazioni in inglese ${ }^{6}$.

La Commissione europea intende fare in modo che i visitatori del sito possano trovare le informazioni di cui hanno bisogno, in una lingua che comprendono, anche se non è la loro lingua madre. Il nostro obiettivo è trovare un ragionevole equilibrio fra le lingue diverse parlate dai cittadini dell'UE e considerazioni di ordine pratico, come i costi di traduzione. Alcuni tipi di contenuti, come la legislazione, sono sempre disponibili in tutte le lingue dell'UE. Altri potrebbero essere disponibili soltanto in una lingua o in una combinazione di lingue che, in base ai risultati di una ricerca sugli utenti, ci consentiranno di raggiungere il più vasto pubblico possibile nel modo più efficiente possibile. Tutti i contenuti sono pubblicati almeno in inglese, in quanto la ricerca ha dimostrato che l'inglese è la lingua compresa dal 9o\% circa dei visitatori dei nostri siti, come lingua straniera preferita o come lingua materna. Inoltre monitoriamo il comportamento degli utenti per vedere se cercano di visualizzare pagine in una determinata lingua, in modo da chiedere la traduzione delle pagine più richieste.

È lecito essere scettici sui dati riportati nel testo sopra citato. In primo luogo, vi è probabilmente un effetto detto di 'autoselezione'. Essendo l'inglese l'unica lingua in cui tutti i contenuti del sito della Commissione sono disponibili, è probabile che gli utenti potenzialmente interessati a visitare il sito siano proprio persone che già conoscono questa lingua.

In secondo luogo, stando ai dati ufficiali sulle competenze linguistiche degli europei pubblicate da Eurostat, l'agenzia europea di statistica, la percentuale della popolazione dell'UE a 27 stati membri che dichiara di non conoscere l'inglese si attesta fra il $51 \%$ e il $56 \%$ a seconda dei campioni (Gazzola 2or6b). Il resto della popolazione ha delle competenze per lo più elementari o intermedie, mentre la percentuale di coloro che dichiarano di conoscere molto bene l'inglese come lingua straniera non 
supera l's\% della popolazione europea. Si tratta per lo più di residenti appartenenti alla fascia della popolazione più istruita e ad alto reddito. L'inglese inoltre è attualmente la lingua materna di meno del I\% della popolazione dell'Unione orfana del Regno Unito. Si tratta per lo più di irlandesi o di britannici residenti nei paesi dell'Unione. Il caso irlandese è interessante. La Repubblica d'Irlanda ha due lingue ufficiali, l'inglese e l'irlandese (o gaelico), anch'esso lingua ufficiale dell'Unione, che però è poco usato dagli abitanti del paese con l'eccezione di una piccola percentuale della popolazione residente in alcune zone della costa occidentale note come Gaeltacht. Per un principio di democrazia linguistica è quindi giusto che l'inglese resti una lingua ufficiale dell'Unione, ma è necessario chiedersi quali siano le conseguenze di un suo uso prevalente nella comunicazione dell'Unione rivolta al pubblico.

Facendo quindi la somma di chi parla inglese come lingua materna e chi lo conosce come lingua straniera a un livello molto buono, ovvero il livello necessario per comprendere testi riguardanti il coronavirus o i progetti europei, si arriva a nemmeno il ıo\% della popolazione. Il restante 90\% o non capisce l'inglese o non lo parla bene. Non si vuole qui sminuire il valore delle competenze di livello elementare o intermedio in una lingua seconda. Tuttavia, l'utilizzo prevalente dell'inglese in molte pagine della Commissione europea si rivela essere una scelta subottimale dal punto di vista dell'efficacia comunicativa rispetto a una politica plurilinguistica. Infine si crea il paradosso per cui è in media più semplice per un cittadino britannico o australiano avere accesso ai contenuti pubblicati dalla Commissione che per un contribuente europeo non di lingua madre inglese.

Si potrebbe obiettare che si tratta di una situazione destinata a risolversi da sola nell'arco di una generazione, in particolare grazie all'azione dell'istruzione. L'Unione europea raccomanda agli Stati membri di insegnare almeno due lingue straniere fin dall'infanzia7. In pratica peró osserviamo un'incontrastata egemonia dell'insegnamento dell'inglese ${ }^{8}$. Secondo i dati di Eurostat disponibili per l'anno 20I7, nelle scuole primarie dell'Unione a 27 stati membri (escludendo quindi il Regno Unito), l'inglese è insegnato a l' $82 \%$ dei bambini, seguito da francese (5\%) e tedesco (4\%). A livello di scuole secondarie di primo grado, il $98 \%$ dei bambini impara l'inglese, seguito da francese $(33 \%)$, tedesco (23\%), spagnolo (I7\%), russo (6\%) e italiano (I\%). Infine, a livello di istruzione secondaria di secondo grado, il $97 \%$ dei ragazzi impara l'inglese, seguito da spagnolo (26\%), francese (23\%), tedesco (21\%), italiano e russo, entrambi al $3 \%$.

L'apprendimento delle lingue straniere nei sistemi di istruzione pubblica va certamente incoraggiato e rinforzato. Tuttavia, è lecito chiedersi se sia ragionevole attendersi che tutti ragazzi alla fine del percorso scolastico siano in grado di esprimersi senza esitazioni in una lingua che non è la propria lingua materna. I dati ancora una volta ci riportano alla realtà dei fatti. Nel 2019 il Consiglio dell'Unione europea faceva notare che nella scuola secondaria solo un allievo su quattro raggiunge un livello di competenza nella prima lingua straniera studiata che gli permette di sostenere una semplice conversazione $^{\mathrm{IO}}$. In altre parole, molti studiano l'inglese a scuola, ma pochi lo imparano a un livello soddisfacente.

\section{Plurilinguismo e traduzione automatica}

Il Regolamento n. I/58 andrebbe probabilmente aggiornato e adattato alla nuova realtà in cui viviamo, ma è improbabile che il Consiglio dell'Unione europea decida di aprire questo vaso di Pandora. È però possibile ricorrere ad altri accorgimenti e rimedi per promuovere il plurilinguismo nella comunicazione rivolta al pubblico senza rimettere mano alla base legale esistente. Tradurre di 
più può certamente contribuire a questo fine. Il contenimento dei costi di traduzione evocato dalla Commissione europea nella citazione qui sopra è un argomento importante da tenere in considerazione. I dati a disposizione, tuttavia, suggeriscono che i costi di traduzione in realtà sono piuttosto contenuti. Le spese per il multilinguismo nelle istituzioni dell'Unione corrispondono a meno dell'ı\% del bilancio dell'Unione e a meno dello o,or\% del prodotto interno lordo dei paesi membri (Gazzola e Grin 2013).

Non volere aumentare i costi di traduzione è certamente una scelta politica legittima. Ma sarebbe allora opportuno fare tesoro dei recenti prodotti dell'innovazione tecnologica, in particolare alla traduzione automatica (Pym 2013). Grazie allo sviluppo della traduzione automatica neurale, che sfrutta l'intelligenza artificiale e vastissimi corpora di traduzioni umane, i traduttori automatici come DeepL oppure Google Translate offrono servizi di traduzione gratuita di qualità molto migliore rispetto al passato, almeno per quanto riguarda testi di natura informativa che non entrano nello specifico dei diversi linguaggi specialistici.

La traduzione automatica, va detto, è uno degli architravi del nuovo sistema di brevetto unificato europeo proprio per volontà della Commissione europea ${ }^{\mathrm{II}}$. Il brevetto unico adottato in prima battuta nel 2012 entrerà in vigore nel 2022 e sarà disponibile solo in francese, tedesco e inglese. La Commissione giocò la carta della traduzione automatica "di alta qualità" (ipsa dixit) per vincere le resistenze degli spagnoli e italiani che avrebbero voluto includere spagnolo e italiano fra le lingue di procedura del brevetto (Gazzola 2oro).

L'Unione europea, e in particolare la Commissione, potrebbe quindi fare un utilizzo più sistematico della traduzione automatica nella comunicazione attraverso le proprie pagine Internet, abbracciando quindi un modello di comunicazione più plurilingue e inclusiva. Se e quando non è possibile tradurre tutte le pagine del suo sito, ad esempio, i comunicati e alcuni documenti non legalmente vincolati, la Commissione potrebbe però pubblicare $\mathrm{i}$ testi in un formato elettronico predefinito facilmente trasferibile in un sistema di traduzione automatica. Ad esempio, si potrebbero evitare di pubblicare testi in formati difficili da copiare e incollare (pensiamo ai PDF formattati o scansioni).

Questa scelta presenterebbe un secondo vantaggio. Non soltanto le traduzioni, seppure imperfette, sarebbero subito fruibili in attesa delle traduzioni umane (le migliori e le uniche fedeli, va detto), ma i funzionari europei sarebbero liberi di redigere e pubblicare più documenti in lingue diverse dall'inglese. Non importa più a quel punto se un comunicato sarà pubblicato in prima battuta solo in tedesco, oppure se le linee guida di un progetto o un bando saranno disponibili temporaneamente solo in francese, perché gli utenti potranno tradurre automaticamente il testo in questione nella loro lingua materna, incluso ovviamente l'inglese.

\section{Nota bibliografica:}

- Chiti-Gualdo 2008: Edoardo Chiti, Riccardo Gualdo (a cura), Il regime linguistico dei sistemi comuni europei. L'Unione fra multilinguismo e monolinguismo. Milano, Giuffe, 2008.

- Commissione europea 20I2: First European survey on language competences, Bruxelles, Commissione europea, 2012.

- Di Stefano 2020: Paolo Di Stefano, Anche nelle emergenze la Commissione Ue parla solo inglese, "Corriere della Sera", 26 febbraio 2020.

- Eurydice 20I7: Cifre chiave dellinsegnamento delle lingue a scuola in Europa, Bruxelles, 
Commissione europea, 2017.

- Gazzola 2006: Michele Gazzola, La gestione del multilinguismo nell'Unione europea, in Augusto Carli (a cura) Le sfide della politica linguistica di oggi: fra la valorizzazione del multilingualismo migratorio locale e le istanze del plurilinguismo europeo, Milano, Franco Angeli, 20o6, pp. I7-II7.

- Gazzola 2oro: Michele Gazzola, Quali lingue per il brevetto dell'Unione europea? Un'analisi economica, "La Crusca per Voi", 4I, 20IO, pp. 7-IO.

- Gazzola 20r4: Michele Gazzola, Partecipazione, esclusione linguistica e traduzione: Una valutazione del regime linguistico dell'Unione europea, "Studi Italiani di Linguistica Teorica e Applicata", 43 (2), 2014, pp. 227-264.

- Gazzola 2or6a: Michele Gazzola, Documenti e orientamenti dell'Unione europea in materia di multilinguismo, in Paolo Caretti, Paolo e Giuseppe Mobilio (a cura), La lingua come fattore di integrazione sociale e politica, pp. 99-г31, Torino, Giappichelli, 2016.

- Gazzola 2or6b: Michele Gazzola, Research for Cult Committee - European Strategy for Multilingualism: Benefits and Costs, PE 573.460, Brussels, European Parliament, 2016.

- Gazzola-Grin 2013: Michele Gazzola, François Grin, Is ELF more effective and fair than translation? An evaluation of the EU's multilingual regime, "International Journal of Applied Linguistics", 23 (I), 2013, pp. 93-107.

- Kruse-Ammon 20r8: Jan Kruse, Ulrich Ammon, The language planning and policy for the European Union and its failures, in Chua, Catherine Siew Kheng (a cura), Unintended Language Planning in a Globalising World: Multiple Levels of Players at Work, Berlin, De Gruyter Open, 2018, pp. 39-56.

- Mediatore europeo 20r9: Multilinguismo nelle istituzioni dell'UE-Relazione sulla consultazione pubblica, Bruxelles, Mediatore europeo 2019.

- Pingel 20r5: Isabelle Pingel (a cura), Le multilinguisme dans l'Union européenne, Parigi, Pedone, 2015 .

- Pym 2013: Anthony Pym, Translation as an Instrument for Multilingual Democracy, "Critical Multilingualism Studies", I (2), 2013, pp. 78-95.

- Van der Jeught 2015: Stefaan Van der Jeught, EU Language Law, Groningen, Europa Law Publishing, 2015.

- Ziller 2020: Jacques Ziller, Perché linglese rimane una delle lingue ufficiali e di lavoro dell'Unione europea nonostante la Brexit, "Eurojus", (I), 2020, pp. 9I-94.

\footnotetext{
I Si vada l'interrogazione con richiesta di risposta scritta prioritaria alla Commissione europea P-oor950/202I presentata da due deputati europei (Elisabetta Gualmini e Massimiliano Smeriglio, entrambi di Alleanza Progressista dei Socialisti e dei Democratici), e l'Interrogazione a risposta orale al Presidente del Consiglio dei ministri italiano (Atto n. 3-0245o, 22 aprile 202I, seduta n. 319, presentata dalle senatrici Laura Garavini e Nadia Ginetti). Una situazione simile si è ripetuta nel 2014 con la precedente edizione di Erasmus+ (vedi interrogazione scritta E-000507/I4 presentata dagli eurodeputati Giancarlo Scottà e Lorenzo Fontana alla Commissione: "Mancata traduzione del programma Erasmust").

${ }^{2}$ Per una panoramica rimando a Gazzola 2016a.

3 Rimando a Kruse-Ammon 2018, Van der Jeught 2015, Chiti-Gualdo 2008, e Gazzola 2006.

${ }^{4}$ Rimando a diversi contributi in Pingel 2015.

5 Si veda a tal riguardo la lista di documenti citati nel sito della Camera dei Deputati italiana: https://bit.ly/3zTgXFt.

${ }^{6}$ Si veda https://ec.europa.eu/info/language-policy_it.

7 Si vedano le conclusioni del Consiglio Europeo di Barcellona i5 e i6 marzo 2002. SN ioo/o2.

${ }^{8}$ Si veda la pagina di Eurostat What languages are studied the most in the EU?, pubblicata il 25 settembre 2020.

9 Percentuali arrotondate all'unità. Si veda https://bit.ly/3vLwAbO. Si veda anche Eurydice (20I7).
} 
Io Si veda la Raccomandazione del Consiglio del 22 maggio 2019 su un approccio globale allinsegnamento e all'apprendimento delle lingue. Si veda anche Commissione europea 2012.

${ }^{\text {II }}$ Regolamento (UE) N. I260/20I2 del Consiglio del I7 dicembre 20 I2 relativo all'attuazione di una cooperazione rafforzata nel settore dell'istituzione di una tutela brevettuale unitaria con riferimento al regime di traduzione applicabile (Gazzetta ufficiale dell'Unione europea, L 36r/89).

\section{Cita come:}

Michele Gazzola, Può la traduzione automatica favorire il plurilinguismo nell'Unione europea post-Brexit?, "Italiano digitale", XVIII, 2021/3 (luglio-settembre)

DOI: $10.35948 / 2532-9006 / 2021.10588$

Copyright 2021 Accademia della Crusca

Pubblicato con licenza creative commons CC BY-NC-ND 